\title{
Comparaçáo do Equilíbrio de Crianças com Paralisia Cerebral e Crianças com Desenvolvimento Motor Normal
}

\author{
Comparison of the Balance of Children with Cerebral Palsy and Children with Normal Motor Development \\ Luciana Nakaya ${ }^{1}$, Carla Mazzitelli², Cristina dos Santos Cardoso de Sá3
}

\begin{abstract}
RESUMO
A Paralisia Cerebral (PC) refere-se ao déficit neuromuscular causado por uma lesão não-progressiva do encéfalo imaturo resultando em uma variedade clínica, porém apresentando em comum o déficit motor e de integridade sensorial. Objetivo. O objetivo deste estudo foi comparar descritivamente o desempenho de equilíbrio entre crianças com PC hemiparéticas espásticas classificadas em nível I pelo Gross Motor Function Classification System (GMFCS) e crianças em desenvolvimento normal (DN) de mesma idade, por meio da posturografia dinâmica. Resultados. Como resultados encontramos similaridade entre os grupos tanto para as variáveis de estabilidade quanto de ajuste postural, porém pode-se perceber maior dificuldade das crianças com PC em manter e recuperar o equilíbrio no eixo anteroposterior. O grupo PC em algumas condiçóes apresentou melhor desempenho quando comparados as crianças com desenvolvimento normal. Conclusão. Concluímos que as similaridades encontradas devem-se ao fato de ambos os grupos apresentarem-se ainda em fase neuronal de maturação do controle postural, e que as crianças com PC apresentaram maior oscilaçáo e deslocamento do centro de pressão no sentido anteroposterior.
\end{abstract}

Unitermos. Paralisia Cerebral, Equilíbrio Postural, Fisioterapia.

Citaçáo. Nakaya L, Mazzitelli C, Sá CSC. Comparação do Equilíbrio de Crianças com Paralisia Cerebral e Crianças com Desenvolvimento Motor Normal.

\begin{abstract}
Cerebral palsy (CP) refers to neuromuscular deficit caused by a nonprogressive lesion of the immature brain resulting in a clinical variety, but presenting a common motor and sensory integration deficit. Objective. The aim of this descriptive study was to compare the performance of balance between hemiparetic spastic children with CP classified at Gross Motor Function Classification System (GMFCS) level I and normal development children in the same age by dynamic posturography. Results. As a result, we find similarity between both groups for the variables of postural stability and adjustment, and we could see greater difficulty of children with CP to maintain and restores balance in the anteroposterior axis, although the CP group under some conditions, performed better when compared to children in normal development. Conclusion. We conclude that the similarities found are due to the fact that both groups were still in neuronal maturation of postural control, and that children with CP had greater sway and displacement of the center of pressure in the anteroposterior direction.
\end{abstract}

Keywords. Cerebral Palsy, Postural Balance, Physical Theraphy Specialty.

Citation. Nakaya L, Mazzitelli C, Sá CSC. Comparison of the Balance of Children with Cerebral Palsy and Children with Normal Motor Development.
Estudo realizado na Universidade Metodista - Sáo Paulo em parceria com a Universidade Federal de Sáo Paulo - Campus Baixada Santista, Santos-SP, Brasil.

1.Fisioterapeuta, especialista em fisioterapia neurológica, São Paulo-SP, Brasil. 2.Fisioterapeuta, Profa. Doutora, Curso de Fisioterapia Universidade Metodista - São Paulo, São Bernardo do Campo-SP, Brasil.

3.Fisioterapeuta, Profa. Doutora, Departamento de Ciências do Movimento Humano - UNIFESP- Campus Baixada Santista, Santos-SP, Brasil.
Endereço para correspondência: Profa. Dra. Cristina dos Santos Cardoso de Sá Av. D. Ana Costa, 95 - Vila Mathias CEP 116060-001, Santos-SP, Brasil. 


\section{INTRODUÇÃO}

A Paralisia Cerebral (PC) é caracterizada por apresentar múltiplos distúrbios de ordem permanente, provocando acometimento do movimento e postura, os quais resultam limitaçôes das atividades diárias ${ }^{1-4}$.

Observa-se nas crianças com PC atrasos na aquisição de habilidades manipulatórias e de mobilidade, que interferem na qualidade de vida, pois prejudicam uma vida independente ${ }^{5-7}$. Crianças com PC apresentam ativação de músculos antagonistas antes dos agonistas durante a movimentação, lentificação no recrutamento dos motoneurônios, problemas de modulação na ordem de recrutamento muscular, e alteração do tônus muscular que provocam atrasos no início do movimento. Alterações do sistema musculoesquelético geradas por compensaçôes, como amplitude de movimento restrita de tornozelos, joelhos e quadril, contraturas musculares e fraqueza muscular favorecem um controle postural deficitário. Assim, essas limitações afetam a função postural, e, portanto o equilíbrio dessas crianças ${ }^{8-13}$.

A conservação do equilíbrio, durante a movimentação ou manutenção de qualquer postura, requer que forças que controlam a posição do corpo no espaço sejam geradas de acordo com o grau de instabilidade. Isso significa que a perturbação da estabilidade é combatida por uma resposta muscular de amplitude adequada e, para que isso ocorra, é necessária uma sequência de ativação muscular, que resulte na recuperação do equilíbrio, como também da interação entre os componentes dos sistemas visual, somatossensorial e vestibular ${ }^{10}$.

O déficit de equilíbrio em crianças com PC vem sendo muito estudado. Alguns estudos empregam a plataforma de força e mensuram a área e o deslocamento do centro de pressão do indivíduo e a velocidade deste deslocamento. Estudo envolvendo crianças com PC espástica, hemiparéticas e diparéticas, revelou que estas apresentam desequilíbrio após pequeno deslocamento da plataforma, maior deslocamento total do centro de pressão e do tempo de recuperaçáo do equilíbrio do que em crianças da mesma idade sem alteraçôes do desenvolvimento motor ${ }^{8}$.

Nota-se que o controle postural é crítico para a aquisição das habilidades motoras, bem como para a produção de movimentos voluntários coordenados e funcionais. Assim, as disfunçôes deste controle são alteraçôes importantes, e responsáveis por baixo desempenho nas atividades funcionais e de vida diária de crianças com PC.

A hipótese deste estudo é que crianças com PC apresentem maior área de deslocamento do centro de pressão, maior velocidade do centro de pressáo, que revela alterações dos ajustes posturais, em relação as crianças com desenvolvimento motor normal. Este conhecimento é fundamental para a compreensão do controle postural nas crianças com PC, o que auxiliará na fundamentação do planejamento terapêutico específico, como também na escolha da abordagem terapêutica, visando maior funcionalidade e independência de crianças com PC.

O presente estudo objetivou comparar o equilíbrio de crianças com Paralisia Cerebral e crianças sem alterações do desenvolvimento motor, e verificar a participação de cada informação sensorial no ajuste postural das crianças com Paralisia Cerebral.

\section{MÉTODO}

\section{Participantes}

Foram avaliadas 12 crianças, seis com diagnóstico de PC apresentando quadro de hemiparesia espástica em atendimento fisioterapêutico, e seis crianças sem alteração do desenvolvimento motor. Os grupos foram pareados de acordo com a idade e gênero.

Como critério de inclusão para o grupo de crianças com PC considerou-se: 1) Diagnóstico de PC espástico hemiparético; 2) Classificação de acordo com Gross Motor Function Classification System (GMFCS) em nível I; 3) Estar em acompanhamento fisioterapêutico. Para as crianças sem alteração do desenvolvimento motor considerou-se: 1) não ter nenhuma anormalidade de ordem musculoesquelética, ter a mesma idade e possuir o mesmo gênero das crianças do grupo PC.

Como critérios de exclusão considerou-se: 1) a não concordância dos pais ou responsáveis quanto à participação de seu filho no estudo; 2) possuir o diagnóstico de PC e não estar em atendimento fisioterapêutico. Para as crianças sem alteraçáo do desenvolvimento motor considerou-se: 1) a não concordância dos pais ou responsáveis quanto à participaçáo de seu filho no estudo; 2) estar realizando fisioterapia por problemas de ordem musculoesquelética.

A Tabela 1 ilustra a caracterização dos participantes do estudo. A pesquisa foi conduzida após aprovação pelo 
Comitê de Ética e Pesquisa da Universidade Metodista de São Paulo (CEP 1747/10), em obediência a Resolução 196/96 sobre "Pesquisa Envolvendo Seres Humanos", do Conselho de Saúde do Ministério de Saúde.

Tabela 1

Caracterização dos participantes

\begin{tabular}{cccc}
\hline $\begin{array}{c}\text { Número de } \\
\text { Identificaçáo da } \\
\text { criança }\end{array}$ & Idade & Diagnóstico & Gênero \\
\hline $\mathbf{1}$ & 6 ANOS & PC-HEMI E & F \\
$\mathbf{2}$ & 6 ANOS & DN & F \\
$\mathbf{3}$ & 9 ANOS & PC HEMI E & F \\
$\mathbf{4}$ & 9 ANOS & DN & F \\
$\mathbf{5}$ & 10 ANOS & PC HEMI D & F \\
$\mathbf{6}$ & 10 ANOS & DN & F \\
$\mathbf{7}$ & 6 ANOS & PC HEMI E & M \\
$\mathbf{8}$ & 6 ANOS & DN & M \\
$\mathbf{9}$ & 10 ANOS & PC HEMI D & M \\
$\mathbf{1 0}$ & 10 ANOS & DN & $\mathrm{M}$ \\
$\mathbf{1 1}$ & 12 ANOS & PC HEMI D & $\mathrm{M}$ \\
$\mathbf{1 2}$ & 12 ANOS & DN & $\mathrm{M}$ \\
\hline
\end{tabular}

\section{Equipamentos e Procedimentos}

As avaliaçóes foram realizadas no Laboratório de Psicofarmacologia, Psicopatologia Experimental e Terapêutica Psiquiátrica do Instituto de Psiquiatria do Hospital das Clínicas da Faculdade de Medicina da Universidade de São Paulo (LIM 23 - HC/FMUSP).

A avaliaçáo de cada paciente foi realizada por meio do Teste de Organização Sensorial Modificado (OSM) da Posturografia Dinâmica Computadorizada (Pro Balance Master - NeuroCom Inc. Oregon, EUA), que forneceu dados sobre o equilíbrio dos participantes.

$\mathrm{O}$ Teste OSM consiste em um procedimento não invasivo que determina o desempenho do equilíbrio postural em quatro diferentes condiçóes sensoriais. Este teste avalia o equilíbrio sob a combinação de condiçóes sensoriais visuais (olhos abertos e olhos fechados) e da superfície de apoio (plataforma fixa e plataforma móvel). Nas condições em que o teste exigia a manutenção dos olhos fechados, as crianças foram vendadas, a fim de garantir que a visão não estava sendo utilizada.

As crianças foram avaliadas descalças, usando rou- pas confortáveis e supervisionadas pelo examinador. Foram instruídas a ficar em pé sobre a plataforma com os braços ao longo do corpo. Os pés foram posicionados na plataforma pelo examinador de acordo com a padronizaçâo indicada pelo equipamento, que se baseia na altura do indivíduo. Um molde da base de sustentaçấo de cada criança foi feito em cartolina, a fim de garantir o mesmo posicionamento, caso fosse necessária à correção da base de sustentação nas diferentes condições de teste. Nesta posição, três tentativas de 20 segundos foram coletadas a $100 \mathrm{~Hz}$ de frequência de amostragem, em quatro condiçôes de teste: plataforma fixa/olhos abertos (condição 1); plataforma fixa/olhos fechados (condição 2); plataforma móvel/olhos abertos (condição 3) e plataforma móvel/ olhos fechados (condição 4), sempre respeitando esta sequência.

As coordenadas do centro de pressão (COP) foram calculadas no programa de computador MATLAB ${ }^{\circ}$, a partir dos dados obtidos por meio da plataforma de força COPy (deslocamento no sentido anteroposterior) e o COPx (deslocamento no sentido medio-lateral).

O desempenho da estabilidade postural foi avaliado por meio das variáveis: área (quanto maior o valor da medida, maior a área de excursão indicando maior oscilação, e portanto maior instabilidade postural), COPx, COPy, e o desempenho do ajuste postural por meio das variáveis: VMx (velocidade de deslocamento no sentido médio-lateral) e Vmy (Velocidade de deslocamento no sentido anteroposterior), as quais foram obtidas para cada uma das três tentativas das condiçóes avaliadas.

\section{Análise Estatística}

Foi realizada uma análise descritiva para cada comparação entre criança PC e criança com desenvolvimento normal (sem alteraçáo), considerando cada variável (área, COPx, COPy, VMx e VMy) em cada condição de teste, ou seja, plataforma fixa com olhos abertos (condiçáo 1) e fechados (condição 2), plataforma móvel com olhos abertos (condição 3) e fechados (condição 4).

\section{RESULTADOS}

Os resultados apresentados referem-se aos parâmetros da estabilidade avaliados por meio das variáveis: área, COPx, COPy, e ajuste postural avaliados por meio das 
variáveis: VMx e VMy. Estes são apresentados comparando o equilíbrio das crianças sem alteraçóes do desenvolvimento motor e das crianças com hemiparesia espástica decorrentes de PC classificadas de acordo com o GMFCS em nível I. Nas Tabelas de 2 a 4, as crianças foram indicadas por números de 1 a 12 . O número ímpar indica crianças com PC, e o número par indica crianças sem alterações do desenvolvimento motor.

\section{Estabilidade}

A Tabela 2 ilustra que a área de projeção do centro de pressão das crianças 1, 3, 5 e 7 com $\mathrm{PC}$ e crianças 2, 4, 6 e 8 sem alteraçóes do desenvolvimento são similares nas diferentes condições testadas. $\mathrm{O}$ comportamento do equilíbrio tanto das crianças com PC quanto daquelas sem alteraçáo do desenvolvimento motor é similar de acordo com a complexidade de cada condição testada. Porém, a criança 8 , sem alteração do desenvolvimento, apresentou valores superiores a criança 7 (PC) na condição 4, considerada a mais complexa.

Por outro lado a criança 9 (PC) apresentou valores superiores apenas na condição 4 quando comparada a criança 10 (sem alteraçáo), o que era esperado por ser esta a condição de manutenção do equilíbrio mais complexa. Já a criança 11 (PC), quando comparada à criança 12 (sem alteração), apresentou valores superiores nas condiçôes 1, 2 e 4, indicando maior área de projeção do centro de pressão nestas condições de teste, o que é esperado por apresentar uma lesão no SNC, mas curiosamente apresentaram valores similares na condição 3 (Tabela 2 ).

A Tabela 3 revela o deslocamento do centro de pressão no eixo látero-lateral (COPx) nas quatro condiçôes testadas. De modo geral, houve similaridade neste deslocamento entre as crianças com PC e as crianças sem alteração do desenvolvimento motor, como pode ser observado nos valores médios obtidos pelas crianças com PC, 3, 5 e 11 e as crianças 4, 6 e 12 sem alteraçáo do desenvolvimento, revelando que o controle postural no sentido látero-lateral destas crianças é similar.

Curiosamente, a criança 6 que possui desenvolvimento motor normal, apresentou valores médios superiores aos da criança 5 em todas as condiçōes testadas (Tabela 3). Por outro lado, as crianças 1 e 9 com PC apresentaram valores médios superiores nas condiçóes 3 e 4 quando comparadas às crianças 2 e 10 , sem alteração, o que era esperado por serem tarefas mais complexas (Tabela 3 ).

A Tabela 3 ainda revela o deslocamento do centro de pressão no eixo anteroposterior (COPy) nas quatro condições de teste. De modo geral, as crianças sem alte-

Tabela 2

Média e desvio padrão de cada criança nas diferentes condiçōes testadas (as crianças que receberam número impar são crianças com PC. As crianças que receberam número par são crianças com desenvolvimento motor normal)

\begin{tabular}{cccccc}
\hline Crianças & Idades & condição 1 & condiçáo 2 & condiçáo 3 & condição 4 \\
\hline 1 & 6 anos & $2,20 \pm 0,21$ & $2,49 \pm 0,07$ & $2,60 \pm 0,09$ & $6,44 \pm 4,342$ \\
2 & 6 anos & $1,69 \pm 0,34$ & $2,04 \pm 0,20$ & $3,50 \pm 1,53$ & $4,71 \pm 1,64$ \\
3 & 9 anos & $0,46 \pm 0,17$ & $1,01 \pm 0,41$ & $3,21 \pm 1,77$ & $8,67 \pm 2,73$ \\
4 & 9 anos & $2,52 \pm 0,35$ & $2,70 \pm 3,19$ & $2,59 \pm 0,24$ & $10,17 \pm 1,50$ \\
5 & 10 anos & $0,64 \pm 0,09$ & $1,58 \pm 0,78$ & $3,79 \pm 2,46$ & $6,26 \pm 1,41$ \\
6 & 10 anos & $1,87 \pm 0,96$ & $3,83 \pm 1,74$ & $4,24 \pm 0,87$ & $6,05 \pm 4,64$ \\
7 & 6 anos & $0,78 \pm 0,47$ & $0,93 \pm 0,60$ & $2,74 \pm 1,85$ & $0,83 \pm 1,63$ \\
8 & 6 anos & $2,51 \pm 0,75$ & $1,88 \pm 0,48$ & $4,28 \pm 1,65$ & $4,50 \pm 0,71$ \\
9 & 10 anos & $2,58 \pm 0,76$ & $2,34 \pm 0,90$ & $5,31 \pm 2,73$ & $16,83 \pm 7,96$ \\
10 & 10 anos & $4,89 \pm 1,71$ & $1,43 \pm 3,19$ & $2,59 \pm 0,78$ & $35,01 \pm 9,82$ \\
11 & 12 anos & $0,17 \pm 0,00$ & $0,32 \pm 0,09$ & $2,17 \pm 1,50$ & $6,26 \pm 2,92$ \\
12 & 12 anos & $4,88 \pm 2,44$ & $3,49 \pm 2,03$ & $2,07 \pm 0,49$ & $11,47 \pm 6,00$ \\
\hline
\end{tabular}


ração do desenvolvimento motor apresentam valores médios de deslocamento similares ou superiores as crianças com PC, principalmente nas condiçóes 3 e 4, em que a informação somatossensorial não é confiável, indicando maior oscilação do centro de pressão no sentido anteroposterior das crianças com PC.

\section{Ajustes Posturais}

A Tabela 4 revela a velocidade média da excursão do centro de pressão no eixo látero-lateral $(\mathrm{VMx})$ nas quatro condiçóes testadas. Em seis das crianças participantes, três com PC (crianças 3, 5 e 11) e três sem alteraçóes do desenvolvimento motor (crianças 4, 6 e 12) os valores médios da velocidade no sentido látero-lateral foram similares, o que indica que os ajustes posturais no sentido látero-lateral de crianças com alteração do desenvolvimento e com PCs são parecidos.

No entanto, na comparação entre a criança 1 e 2 pode-se observar que a criança sem alteração do desenvolvimento (criança 2) apresenta valores médios superiores na condição 4 (Tabela 4). Já na comparação entre a criança 7 e 8 ; e 9 e 10 as crianças com PC apresentam valores médios superiores na condição 4 .

Por fim, a Tabela 4 ainda revela a velocidade média de excursão do centro de pressão no eixo anteroposterior (VMy) nas quatro condiçốes testadas. De modo geral, as crianças com PC apresentam maior velocidade de oscilação do centro de pressão no sentido anteroposterior nas condições 3 e 4, condições em que a plataforma oscila, indicando que há uma diferença em relação aos ajustes

Tabela 3

Média e desvio padrão das variáveis COPx (deslocamento do centro de pressáo no sentido látero-lateral) e COPy (deslocamento do centro de pressáo no sentido anteroposterior de cada criança nas diferentes condiçôes testadas (as crianças que receberam número impar são crianças com PC. As crianças que receberam número par são crianças com desenvolvimento motor típico)

\begin{tabular}{|c|c|c|c|c|c|c|}
\hline Crianças & Idades & Variável & condição 1 & condiçáo 2 & condição 3 & condição 4 \\
\hline \multirow[t]{2}{*}{1} & 6 anos & COPx & $45,53 \pm 9,75$ & $49,78 \pm 1,57$ & $85,66 \pm 22,55$ & $129,33 \pm 17,22$ \\
\hline & & COPy & $50,07 \pm 0,63$ & $60,85 \pm 3,64$ & $112,96 \pm 25,83$ & $156,09 \pm 31,76$ \\
\hline \multirow[t]{2}{*}{2} & 6 anos & $\mathrm{COPx}$ & $46,54 \pm 0,04$ & $50,56 \pm 0,79$ & $51,03 \pm 0,06$ & $78,21 \pm 1,52$ \\
\hline & & COPy & $46,83 \pm 0,75$ & $55,95 \pm 0,34$ & $65,82 \pm 4,48$ & $130,04 \pm 32,17$ \\
\hline \multirow[t]{2}{*}{3} & 9 anos & COPy & $42,51 \pm 21,69$ & $36,23 \pm 2,32$ & $46,76 \pm 1,95$ & $62,67 \pm 6,80$ \\
\hline & & COPx & $31,99 \pm 0,97$ & $32,77 \pm 4,78$ & $40,94 \pm 6,64$ & $122,77 \pm 8,89$ \\
\hline \multirow[t]{2}{*}{4} & 9 anos & COPx & $22,63 \pm 0,99$ & $22,49 \pm 5,10$ & $23,18 \pm 2,22$ & $30,82 \pm 42,70$ \\
\hline & & COPy & $25,01 \pm 1,36$ & $24,69 \pm 4,03$ & $28,26 \pm 3,74$ & $110,72 \pm 19,51$ \\
\hline \multirow[t]{2}{*}{5} & 10 anos & COPx & $30,03 \pm 11,17$ & $33,72 \pm 3,10$ & $38,72 \pm 2,06$ & $54,14 \pm 13,52$ \\
\hline & & COPy & $34,38 \pm 3,84$ & $46,03 \pm 2,41$ & $67,69 \pm 11,57$ & $111,72 \pm 44,50$ \\
\hline \multirow[t]{2}{*}{6} & 10 anos & COPx & $38,88 \pm 1,82$ & $42,61 \pm 1,68$ & $47,06 \pm 5,17$ & $63,57 \pm 1,98$ \\
\hline & & COPy & $41,05 \pm 3,63$ & $46,85 \pm 1,71$ & $61,62 \pm 2,90$ & $99,32 \pm 9,74$ \\
\hline \multirow[t]{2}{*}{7} & 6 anos & COPx & $96,68 \pm 11,17$ & $95,18 \pm 7,11$ & $135,94 \pm 17,65$ & $118,88 \pm 8,43$ \\
\hline & & COPy & $104,07 \pm 6,11$ & $104,55 \pm 4,02$ & $200,21 \pm 12,69$ & $182,63 \pm 19,39$ \\
\hline \multirow[t]{2}{*}{8} & 6 anos & COPx & $37,14 \pm 2,87$ & $39,92 \pm 1,93$ & $51,23 \pm 4,88$ & $70,47 \pm 5,84$ \\
\hline & & COPy & $43,01 \pm 3,44$ & $45,54 \pm 0,82$ & $73,05 \pm 11,18$ & $131,27 \pm 19,07$ \\
\hline \multirow[t]{2}{*}{9} & 10 anos & COPx & $32,35 \pm 1,55$ & $34,56 \pm 1,95$ & $44,08 \pm 2,60$ & $113,04 \pm 28,33$ \\
\hline & & COPy & $35,03 \pm 1,69$ & $44,15 \pm 2,37$ & $82,15 \pm 18,12$ & $199,81 \pm 29,97$ \\
\hline \multirow[t]{2}{*}{10} & 10 anos & COPx & $32,19 \pm 1,44$ & $31,77 \pm 3,95$ & $37,05 \pm 1,84$ & $53,09 \pm 8,13$ \\
\hline & & COPy & $36,56 \pm 3,63$ & $35,91 \pm 17,70$ & $50,62 \pm 6,81$ & $122,94 \pm 40,64$ \\
\hline \multirow[t]{2}{*}{11} & 12 anos & COPx & $22,55 \pm 2,20$ & $27,55 \pm 0,99$ & $31,97 \pm 2,07$ & $59,36 \pm 6,23$ \\
\hline & & COPy & $24,40 \pm 0,65$ & $28,39 \pm 1,08$ & $40,65 \pm 3,35$ & $127,87 \pm 2,47$ \\
\hline \multirow[t]{2}{*}{12} & 12 anos & COPx & $24,21 \pm 0,35$ & $25,40 \pm 1,25$ & $31,34 \pm 1,15$ & $48,13 \pm 5,94$ \\
\hline & & COPy & $23,49 \pm 3,20$ & $26,73 \pm 0,97$ & $40,12 \pm 3,58$ & $86,10 \pm 20,39$ \\
\hline
\end{tabular}


posturais quando a informação proprioceptiva é abalada.

Vale ressaltar, que as crianças com PC 3 e 5 apresentam valores similares as crianças 4 e 6 sem alteração, revelando que o controle postural são parecidos nestas crianças.

\section{DISCUSSÃO}

O objetivo deste estudo foi comparar descritivamente o equilíbrio entre crianças PC espásticas hemiparéticas classificadas no nível I pelo GMFCS e crianças com desenvolvimento motor normal (DN) de mesma idade e gênero, a fim de verificar o controle postural das mesmas em distintas condiçôes sensoriais (olhos abertos e olhos fechados associados a perturbações externas: plataforma fixa e móvel). Dessa forma pode-se obter dados a respeito da estabilidade e ajustes posturais destas crianças.

Os resultados da área de deslocamento do centro de pressão retratam a estabilidade postural. Desse modo, quanto maior o valor obtido na medida, maior a área de excursão indicando maior oscilação e, portanto, maior instabilidade postural. Na comparação entre crianças com PC e com DN algumas apresentaram similaridade nos valores obtidos em todas as condiçóes testadas, indicando que o comportamento do controle postural em crianças com lesão no sistema nervoso central (SNC) pode evoluir com pequenas sequelas, e estas podem ser imperceptíveis em alguns casos. No entanto, em algumas condiçôes pode-se observar melhor desempenho ora da criança com

Tabela 4

Média e desvio padrão das variáveis VMx (velocidade de deslocamento do centro de pressão no sentido látero-lateral) e VMy (velocidade de deslocamento do centro de pressão no sentido anteroposterior (deslocamento do centro de pressáo no sentido anteroposterior) de cada crianças nas diferentes condiçôes testadas (as crianças que receberam número impar são crianças com PC. As crianças que receberam número par são crianças com desenvolvimento motor normal)

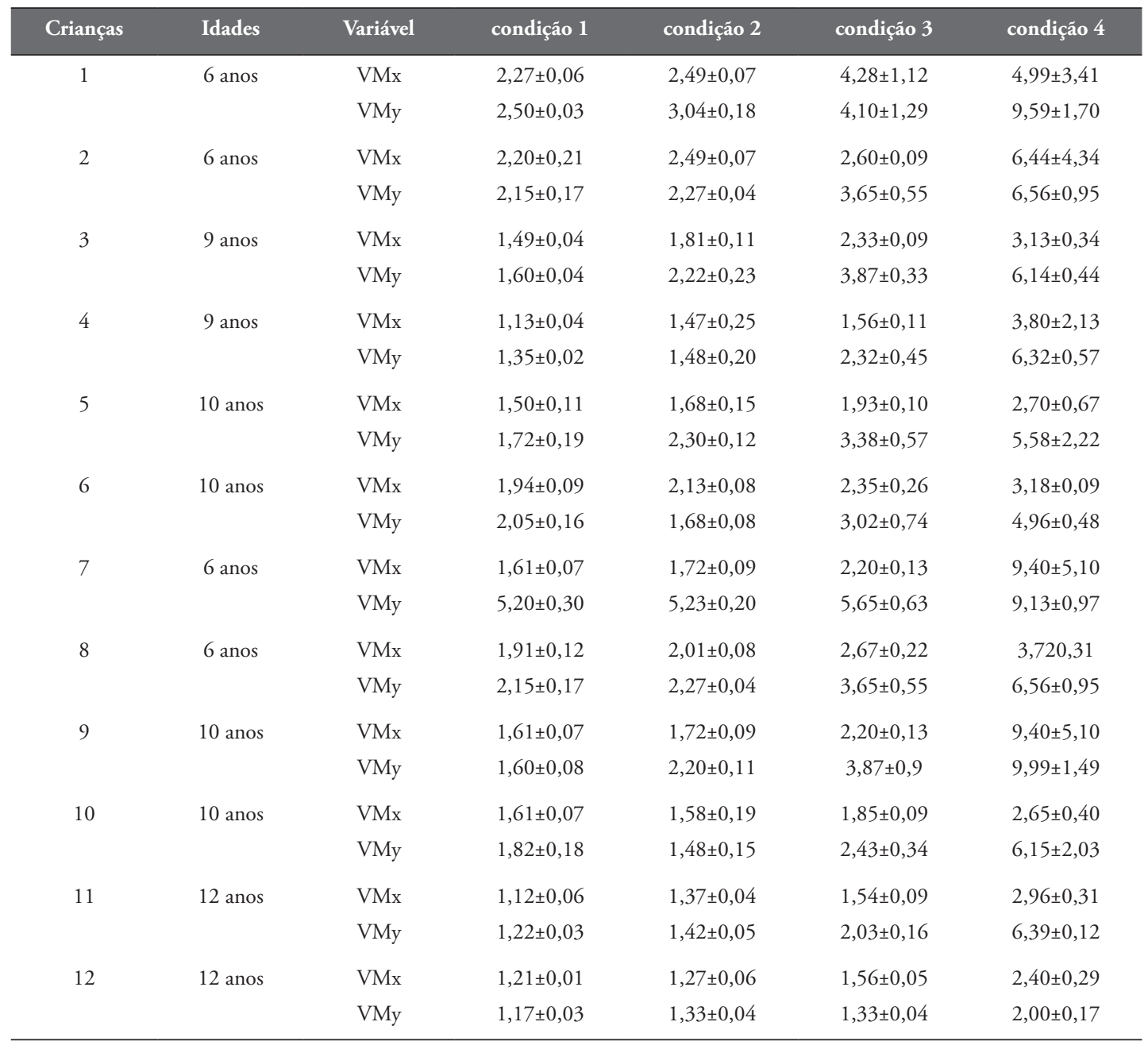


$\mathrm{PC}$, o que não era esperado, e ora da criança com $\mathrm{DN}$, o que era esperado. Isto provavelmente decorre do fato de as crianças com PC realizarem tratamento fisioterapêutico, o qual enfatiza o treinamento do equilíbrio ${ }^{11,14-16}$.

A similaridade de resultados em algumas crianças de ambos os grupos, que também foi encontrada para outras variáveis investigadas, pode decorrer de ambos os grupos, na faixa etária estudada, encontrarem-se em processo maturacional de controle postural, uma vez que o desenvolvimento de movimentos e habilidades motoras resulta da maturação neurológica do $\mathrm{SNC}^{17}$. $\mathrm{O}$ controle postural ainda não está completo aos seis anos, e dura até a adolescência em crianças com desenvolvimento motor normal, o que corrobora com os dados obtidos no presente estudo ${ }^{18}$.

Os resultados do deslocamento do centro de pressão na base de sustentação no sentido látero-lateral, COPx, e no sentido anteroposterior, COPy, que também revelam a estabilidade do equilíbrio foram similares em algumas condições testadas como os resultados obtidos na variável área. Os resultados que concernem ao deslocamento do COPx, indicam que as crianças com PC demonstraram maior dificuldade nas condiçóes 3 e 4 (plataforma móvel/olhos abertos e plataforma móvel/ olhos fechados, respectivamente), consideradas as mais complexas, pois a informação somatossensorial está abalada e o sistema nervoso não pode confiar neste tipo informação para manter a estabilidade. $\mathrm{Na}$ idade de seis anos a criança PC obteve valores médios muito superiores aos da criança com DN, demonstrando maior dificuldade em manter a estabilidade, já que nesta idade a criança é mais dependente das informaçóes visuais para manutenção do equilíbrio $^{14,19}$.

Em relaçáo aos resultados referentes ao deslocamento do COPy, deslocamento no eixo anteroposterior, pode-se observar que no geral os deslocamentos de ambos os grupos foram superiores em termos de valores, demonstrando maior dificuldade em manter a postura neste sentido para ambos os grupos. As condições 3 e 4 continuaram a apresentar as maiores variaçóes, sendo que as crianças com PC obtiveram pior desempenho. Novamente, aos seis anos a criança com PC apresentou valores médios muito superiores a criança com $\mathrm{DN}$, isso pode ter ocorrido também pelo controle muscular alterado, anteriormente descrito; mas também pode demonstrar que estas crianças apresentam maior oscilação corporal no sentido anteroposterior, e portanto maior dificuldade para recuperar a estabilidade. Outro aspecto que pode dificultar a manutenção da postural nesta direção é a imaturidade do sistema vestibular em crianças nesta idade que existe em ambos os grupos testados, porém se exacerba no grupo PC, pois se soma as alteracôes no controle muscular, da possível falta de mobilidade da pelve a qual permitiria a projeção anterior do centro de gravidade corporal $^{8,14,19,20}$.

Estudos mostraram que a maioria das crianças com PC apresenta nível básico de controle postural intacto, porém revelaram disfunção no recrutamento da ordem de músculos posturais e na modulação da contração muscular, e no uso das informaçóes sensoriais. Vale ressaltar que o controle postural é organizado em dois níveis funcionais: o primeiro lida com a geração de padrôes de direção específica para o ajuste postural, sendo que o termo "direção-específica" significa que os músculos dorsais do corpo são primariamente ativados quando o corpo oscila anteriormente, o que acontece, por exemplo, durante o movimento de alcance, enquanto os músculos ventrais do corpo são primariamente ativados em caso de oscilação corporal na direçâo oposta. O segundo nível envolve ajustes desta direção-específica de acordo com fatores multissensoriais (p.e: recrutamento de músculos posturais antagonistas, na organização temporal e na modulação de amplitudes) como as aferências advindas dos sistemas somatossensorial, visual e vestibular ${ }^{19-21}$. Crianças com PC além de apresentarem tônus muscular alterado, tem predileção por um recrutamento muscular de proximal para distal, fato que também interfere na recuperação da estabilidade postural levando-os a obter os valores médios superiores no sentido látero-lateral.

O desenvolvimento da função motora grossa, em algumas crianças classificadas como nível I pelo GMFCS, é muito similar ao desenvolvimento de crianças com desenvolvimento normal ${ }^{22}$. Estes resultados corroboram com os dados obtidos no presente estudo, em que crianças com PC classificadas no nível I do GMFCS, apresentam mínimas alterações no controle postural, que muitas vezes são imperceptíveis nas atividades do dia a dia e durante avaliaçôes, apresentando valores de área, COPx e 
COPy similares aos das crianças sem alteraçóes do desenvolvimento em algumas ou em todas as condiçóes testadas. Outros PCs do presente estudo ainda, devido ao treinamento recebido para melhorar o equilíbrio estático e dinâmico durante a fisioterapia, apresentaram resultados superiores em relação ao controle postural quando comparados as crianças sem alteraçóes do desenvolvimento, as quais possivelmente apresentam hábitos de vida mais sedentários que náo permite o refinamento do controle postural.

Nesse contexto a PC é vista como uma desordem, na qual padróes anormais de movimentação e postura resultam da falta de inibição de centros mais inferiores do sistema nervoso central ${ }^{17}$, o que explica o pior desempenho em algumas condiçóes testadas, em especial a condição 4, considerada a mais complexa, em que há oclusão de visão e movimentação da plataforma, representando condição sensorial alterada e instabilidade, respectivamente.

As variáveis VMx e VMy representam a velocidade média com a qual ocorre o deslocamento do centro de pressão na base de sustentação, no sentido látero-lateral e anteroposterior, respectivamente, ou seja, referem-se a velocidade do ajuste postural em cada direção. Os achados revelam resultados similares entre crianças PC e com DN, o que também é visto nas condiçóes 3 e 4 , as mais difíceis para ambos os grupos realizarem os ajustes.

Aos seis anos pode-se observar que a velocidade do deslocamento do COP é maior tanto no sentido látero-lateral (COPx) quando anteroposterior (COPy). Porém no sentido anteroposterior é evidente que os valores de ambos os grupos foram maiores, demonstrando maior dificuldade no ajuste postural. Interessante, que o ajuste postural, assim como a estabilidade, tendem a melhorar com a idade, em ambos os grupos, confirmando a maturação do controle postural. Isto vai ao encontro das conclusóes obtidas em estudo o qual revela que as estratégias de equilíbrio adotadas pelas crianças e adultos envolvem dois princípios funcionais de organização espacial: o primeiro refere-se a escolha na referência da base de estabilidade, no qual o equilíbrio se baseia, e o segundo refere-se ao domínio gradual dos graus de liberdade das articulaçôes do corpo ${ }^{18}$.

Em crianças é bem estabelecido que pistas visuais sejam importantes no desenrolar do controle de equilíbrio em atividades posturais e locomotoras. Estudos comparando o equilíbrio de crianças com idades entre seis e 14 anos com o equilíbrio de adultos revelaram que este decaiu nas crianças quando a visão foi ocluída, o que confirma os dados obtidos neste estudo. Dos três sistemas sensoriais que governam o controle postural, a propriocepção possui maior influência na detecção da oscilação corporal. Autores $^{8,10}$ reportam que a função do sistema somatossensorial se desenvolve precocemente e chega ao seu nível de maturação entre três e quatro anos, porém em outros estudos crianças de sete a 15 anos apresentaram atraso na maturação da integração proprioceptiva, indicando que o sistema somatossensorial ainda está se desenvolvendo até os 12 anos de idade. Nos adultos, as aferências somatossensoriais são predominantes no controle do equilíbrio, enquanto nas crianças as informaçóes visuais são predominantes, com picos aos 6, 8 e 15 anos de idade ${ }^{18}$. Isto explica a menor estabilidade, e a maior velocidade para o ajuste postural nas condiçóes 3 e 4 em ambos os grupos, e principalmente nas crianças com PC.

Dessa forma pode-se discutir o fato de que, como o sistema somatossensorial não está totalmente desenvolvido na faixa etária da amostra estudada e, principalmente porque as crianças com PC podem apresentar alterações somatossensoriais que influenciam o recrutamento muscular, a oscilação corporal apresenta-se maior no deslocamento e velocidade média do centro de pressão, principalmente no sentido anteroposterior, direção mais difícil de ser estabilizada e ajustada. À medida que a idade das crianças aumenta, a criança com desenvolvimento normal começa a maturar seu sistema somatossensorial para posteriormente utilizá-lo na fase adulta como predominante no reestabelecimento do equilíbrio. Já a criança com PC deve demorar um tempo maior para utilizar este sistema se beneficiando apenas da visão como feedback primário, talvez a informação somatossensorial não se mature por completo mesmo em idades avançadas e não seja um sistema muito utilizado para a população com PC no controle postural.

A idade dos seis anos aparece como a de maior variabilidade de valores e isso pode ser justificado pelo estudo de Schmid et al. ${ }^{23}$ que revelou correlação entre o desenvolvimento do controle de equilíbrio e a idade na 
população pediátrica a partir de dados de posturografia. Em sua conclusão os autores verificaram evidente modificaçáo nas estratégias posturais entre as idades de sete a 11 anos em crianças com desenvolvimento típico. Como a maturação dos sistemas ocorre de forma similar entre as crianças com PC e com desenvolvimento motor normal, provavelmente a criança com PC de seis anos ainda não atingiu as mudanças de estratégias posturais, e pela própria lesão do SNC já apresenta desempenho pior em relação às habilidades motoras de uma criança de mesma idade com desenvolvimento motor normal.

Por fim, outro fator que pode contribuir para o melhor desempenho da criança com PC em determinadas condições é o de que muitas das crianças com PC iniciam a fisioterapia em idades baixas, sendo muito mais estimuladas a ganhar controle postural para desempenhar tarefas que são consideradas simples para crianças com desenvolvimento típico. O objetivo do tratamento fisioterapêutico em crianças com PC é que estas atinjam uma função específica, sendo que o sucesso sempre é dado quando a tarefa é completada e não quando o padrão de movimento normal é atingido, isto é, a fisioterapia facilita o padrão de movimento e controle postural para a prática de atividades funcionais, mesmo que o movimento não seja exatamente igual ao das crianças com desenvolvimento motor normal ${ }^{24,25}$.

O estudo apresenta como limitaçóes o número limitado da amostra, como também a diferença da idade das crianças participantes, o que impossibilita uma análise estatística inferencial, uma vez que a diferença das idades implica em diferença na maturidade do sistema nervoso, e, portanto, no controle postural.

Sugerimos, para estudos posteriores, a ampliaçáo do número amostral, bem como a verificação do comportamento do equilíbrio em crianças com paralisia cerebral classificadas como níveis II e III do GMFCS (Sistema de Classificação da função motora Grossa).

\section{CONCLUSÃO}

$\mathrm{Na}$ comparação de equilíbrio entre crianças PC com nível I pelo GMFCS e crianças com desenvolvimento motor normal os grupos apresentam padrōes de similaridade quanto à estabilidade e ajuste postural, demonstrando níveis neuronais ainda em fase de matura- ção, utilizando primariamente, dentre os sistemas sensório motor, o visual.

As crianças PC apresentaram maior oscilação e deslocamento do centro de pressão no sentido anteroposterior, sendo esta direção a mais difícil de ser controlada, por motivos de ajuste direcional em resposta ao desequilíbrio e recrutamento muscular inadequado.

\section{REFERÊNCIAS}

1.Kerr Graham H, Selber P. Aspects of cerebral palsy. The Journal of Bone \& Joint Surgery Br 2003;85(2):157-66.

http://dx.doi.org/10.1302/0301-620X.85B2.14066

2.Carlberg EB, Hadders-Algra M. Postural Dysfunction in children with cerebral palsy: some implications for therapeutic guidance. Neural Plast 2005;12(2-3):221-8.

http://dx.doi.org/10.1155/NP.2005.221

3.Hatta T, Nishimura S, Inoue K, Yamanaka M, Maki M, Kobayashi N, et al. Evaluating the relationship between the postural adaptation of patients with profound cerebral palsy and configuration of the seating buggy's support surface. Journal of Physiological Anthropology 2007;26(2):217-24.

http://dx.doi.org/10.2114/jpa2.26.217

4.Rosenbaum P, Paneth N, Leviton A. et al. A report: the definition and classification of cerebral palsy April 2006. Dev Med Child Neurol 2007;109:8-14. 5.Saavedra S, Joshi A, Woollacott M, van Donkelaar P. Eye hand coordination in children with cerebral palsy. Exp Brain Res 2009;192:155-65.

http://dx.doi.org/10.1007/s00221-008-1549-8

6.Saavedra S, Woollacott M, van Donkelaar P. Head stability during quiet sitting in children with cerebral palsy: effect of vision and trunk support. Exp Brain Res 2010;201(1):13-23.

http://dx.doi.org/10.1007/s00221-009-2001-4

7.Marinho APS, Souza MAB, Pimentel AM. Desempenho funcional de crianças com paralisia cerebral diparéticas e hemiparéticas. Rev Cienc Méd Biol 2008;7(1):57-66.

8.Wollacott MH, Sumway- Cook A. Postural Dysfunction during standing and walking in children with cerebral palsy: what are the underlying problems and what new therapies might improve balance. Neural Plast 2005;12(2-3):211-9. http://dx.doi.org/10.1155/NP.2005.211

9.Donker SF, Ledebt A, Roerdink M, Savelsbergh GJ, Beek PJ. Children with cerebral palsy exhibit greater and more regular postural sway then typically developing children. Exp Brain Res 2008;184(3):363-70.

http://dx.doi.org/10.1007/s00221-007-1105-y

10.Shumway Cook A, Woollacott MH. Controle Motor: Teoria e aplicaçóes práticas. 2 ed. Barueri: São Paulo; 2003.

11.Mancini MC, Fiuza PM, Rebelo JM, Magalhães LC, Coelho ZAC, Paixão $\mathrm{ML}$ et al. Comparaçáo do desempenho de atividades funcionais em crianças com desenvolvimento normal e crianças com paralisia cerebral. Arq Neuropsiquiatr 2002;60(2-B):446-52.

http://dx.doi.org/10.1590/S0004-282X2002000300020

12.Mota AP, Pereira JS. Influencia da fisioterapia nas alteraçôes motoras em crianças com paralisia cerebral. Fisioterapia Brás 2006;7(3):209-12.

13.Prosser LA, Lee SCK, Vansant AF, Barbe MF, Lauer RT. Trunk and hip 
muscle activation patterns are different during walking in young children with and without cerebral palsy. Phys Ther 2010;90(7):986-97.

http://dx.doi.org/10.2522/ptj.20090161

14.Donker SF, Ledebt A Roerdink M, Savelsbergh GJP, Beek PJ. Children with cerebral palsy exhibit greater and more regular postural sway than typically developing children. Exp Brain Res 2008;184:363-370.

http://dx.doi.org/10.1007/s00221-007-1105-y

15.Dias ACB, Freitas JC, Formiga CKMR, Viana FP. Desempenho funcional de crianças com paralisia cerebral participantes de tratamento multidisciplinar. Fisioterapia e Pesquisa, São Paulo, 2010;17(3):225-9.

http://dx.doi.org/10.1590/S1809-29502010000300007

16.Harbourne RT, Willett S, Kyvelidou A, Deffeyes J, Stergiou N. A Comparison of Interventions for Children With Cerebral Palsy to Improve Sitting Postural Control: A Clinical Trial. Phys Ther 2010;90(12):1881-1898.

http://dx.doi.org/10.2522/ptj.2010132

17.Ketelaar M, Vermeer A, van Petegem-van Beek E, Helders PJ. Effects of a functional therapy program on motor abilities of children with cerebral palsy. Phys Ther 2001;81(9):1534-45.

18.Mallau S, Vaugoyeau M, Assaiante C. Postural strategies and sensory integration: No turning point between childhood and adolescence. PLos One 2010 Sep 29;5(9). pii:e13078.

http://dx.doi.org/10.1371/journal.pone.0013078

19. Heide JC, Begeer C, Fock JM, Otten B, Stremmelaar E, van Eykern LA,
Hadders-Algra M. Postural control during reaching in preterm children with cerebral palsy. Dev Med Child Neurol 2004;46(4):253-66.

http://dx.doi.org/10.1111/j.1469-8749.2004.tb00480.x

20.Wichers M, Hilberink S, Roebroeck ME, van Nieuwenhuizen O, Stam HJ. Motor impairments and activity limitations in children with spastic cerebral palsy: a dutch population based-study. J Rehabil Med 2009;41(5):367-74.

http://dx.doi.org/10.2340/16501977-0339

21.Van der Heide JC, Hadders-Algra M.Postural muscle dyscoordination in children with cerebral palsy. Neural Plast 2005;12(2-3):197-203.

http://dx.doi.org/10.1155/NP.2005.197

22.Beckung E, Carlsson G, Carlsdotter S, Uvebrant P. The natural history of gross motor development in children with cerebral palsy aged 1 to 15 years. Dev Med Child Neurol 2007;49(10):751-6.

http://dx.doi.org/10.1111/j.1469-8749.2007.00751.x

23.Schimid M, Conforto S, Lopez L, Renzi P , D’Alessio T. The development of postural strategies in children: a factorial design study. J Neuroeng Rehabil 2005;30;2:29.

24.Law M, Darrah J, Pollock N, Rosenbaum P, Russel D, Walter SD, et al. Focus on function - a randomized controlled trial comparing two rehabilitation interventions for young children with cerebral palsy. BMC Pediatr 2007;27;7:31.

25.Bartlett DJ, Palisano RJ. A Multivariate model of determinants of motor change for children with cerebral palsy. Phys Ther 2000;80(6):598-614. 